\title{
Inside and out; the activities of senescence in cancer
}

Pedro A. Perez-Mancera ${ }^{1}$, Andrew R. J. Young ${ }^{1}$, and Masashi Narita ${ }^{1}$

${ }^{1}$ Cancer Research UK Cambridge Institute, University of Cambridge, Li Ka Shing Centre, Robinson Way, Cambridge, CB2 ORE, UK.

Correspondence to M.N., e-mail: Masashi.Narita@cruk.cam.ac.uk 


\section{Key points}

- Induction of senescence, a stable state of cell cycle arrest, was originally described in normal cells, but it can also be induced in tumour cells in response to various stresses.

- Senescent cells are metabolically active. In contrast to tumour cells, which typically preferentially use glycolysis in the presence of oxygen to generate energy, senescent cells can exhibit hyperactive mitochondrial respiration (oxidative phosphorylation ) in some contexts.

- Autophagy is activated during senescence, but its importance varies depending on the context.

- The senescence-associated secretory phenotype (SASP) mediates the diverse functionality of senescent cells in an autocrine and paracrine manner, including reinforcing or inducing senescence, activating an immune response, and even promoting tumorigenesis, depending on the context.

- Senescent cells can be eliminated through a SASP-induced immune response, which can involve both innate and adaptive immunity.

- Various triggers such as tissue damage or tumorigenesis-associated stresses can cause stromal cell senescence, which may either facilitate or inhibit tumour progression, depending on the context. 


\begin{abstract}
The core aspect of the senescent phenotype is a stable state of cell cycle arrest. However, this is a disguise that conceals a highly active metabolic cell state with diverse functionality. Both the cell autonomous and the non-cell autonomous activities of senescent cells create spatio-temporally dynamic and context-dependent tissue reactions. For example, the senescence-associated secretory phenotype (SASP) provokes not only tumour suppressive but also tumour promoting responses. Senescence is now increasingly considered as an integrated and widespread component that is potentially important for tumour development, suppression and the response to therapy.
\end{abstract}




\section{Introduction}

Cellular senescence, a state of stable exit from the cell cycle, has often been compared with apoptosis as an intrinsic tumour suppressor mechanism. Various triggers induce either phenotype depending on the cell type and context. Since senescent cells are permanently arrested it was often assumed that senescence was functionally similar to apoptotic cell death, in terms of its effects on tumour suppression and tumour therapy. However, as described in this Review, the view that senescence is an end point of a stress response has been considerably revised over the past decade ${ }^{1}$. Senescence has a heterogeneous phenotype in terms of cellular markers: multiple markers are required to define senescence with confidence. Senescence typically depends on the p53 and RB pathways, but other effector programs confer additional layers of regulation. These include telomere shortening and a persistent DNA damage response, autophagy and other metabolic alterations, altered protein secretion, and epigenetic gene regulation $^{2-4}$. These pathways collectively contribute to the establishment and maintenance of senescent cells and their fitness.

The senescence-associated secretory phenotype (SASP) in particular provides senescent cells with diverse functionality, and the senescence response can have profound cell autonomous and non-cell autonomous activities in vivo ${ }^{5,6}$. Paradoxically, the diverse effects of the SASP include not only tumour suppressive but also tumorigenic aspects. However, there is another twist in the view of the long-term fate of senescent cells, which, similar to apoptotic cells, can be cleared from lesions in some contexts. We have summarised senescence markers and senescence associated effector programs in a recent review ${ }^{4}$. Here, we provide an overview of the different roles that senescence has in the development of cancer. We initially focus on the more established cell autonomous role of senescence as a tumour suppressive mechanism, 
emphasising the emerging metabolic features of senescent cells. We then discuss the non-cell autonomous activities of senescence, including their functionality in both tumour suppressive and tumorigenic contexts and their potential impact on the outcome of cancer treatment. Although the focus of this review is on analysing senescence in cancer, its implications in other biological contexts are also briefly summarised in Box 1.

\section{Senescence in pre-neoplasia}

Cellular senescence was initially described in human diploid fibroblasts (HDFs), which, in contrast to immortalised cells, undergo a 'permanent' proliferative arrest after long-term cell culture in vitro ${ }^{7,8}$. This replicative senescence is mainly attributed to progressive telomere shortening, which provokes a persistent DNA damage response and the activation of tumour suppressive signals, including activation of the p53 and the p16-RB pathways ${ }^{9-11}$. In contrast to humans, mice have long telomeres and adult mouse somatic cells often retain telomerase activity, thereby maintaining telomere length ${ }^{12}$. Loss of telomerase activity in mice has been shown to suppress tumorigenesis in various tumour models ${ }^{13-16}$ and telomere attrition-induced senescence is implicated in these tumour suppressive effects ${ }^{17}$. However, a modest increase in the incidence of spontaneous tumours in highly proliferative cells is evident in these mice, possibly owing to increased chromosomal instability ${ }^{18}$.

The tumour suppressor function of senescence was further reinforced by the discovery of oncogene-induced senescence (OIS). OIS was initially described in culture, where the ectopic expression of oncogenic HRAS-G12V, or its downstream effectors paradoxically led to premature senescence ${ }^{19-21}$. However in 2005 a series of studies $^{22-26}$ identified senescence in several premalignant tumours, both in humans 
and mice. The loss of tumour suppressors, such as $\operatorname{PTEN}^{23}$ (BOX 2), $\mathrm{RB}^{27}$, neurofribromin $1(\mathrm{NF} 1)^{28}$ and inositol polyphosphate-4-phosphatase, type II $(\text { INPP4B })^{29}$, has also been associated with senescence in premalignant tumours.

\section{Therapy-induced senescence}

Senescence can also be induced in response to therapy-induced genotoxic stress. Although therapy-induced senescence (TIS) was first described in tumour cell lines ${ }^{30}$ not long after the discovery of OIS ${ }^{19}$, TIS is a relatively unexplored area. This is perhaps in part because tumour cells often exhibit a mixed phenotype of apoptosis and senescence when treated with chemotherapeutic agents, whereas HDFs are typically resistant to apoptosis and readily undergo senescence. Te Poele et al. ${ }^{31}$ showed that TIS was also evident in human breast cancer samples taken from patients who had undergone pre-operative neoadjuvant chemotherapy. These tumours tended to be positive for senescence-associated- $\beta$-galactosidase (SA- $\beta$-gal) activity and expression of p16, which are widely used markers of senescence, compared with non-treated tumours. Although this study suggested that a senescence-like phenotype can be induced in tumour cells in vivo, the clinical relevance of TIS remains unclear.

The first genetic evidence that senescence induction might represent a worthwhile therapeutic goal was shown in an E $\mu-M y c$ mouse B cell lymphoma model, where $M y c$ is expressed under an immunoglobulin heavy chain enhancer-promoter and therefore restricted to the B-cell compartment. Schmitt et al. ${ }^{32}$ compared chemotherapeutic efficacy in $\mathrm{E} \mu-M y c$ lymphomas with $\mathrm{E} \mu-M y c$; $\operatorname{Tr} 53^{-/-}$lymphomas, and $\mathrm{E} \mu-M y c$ lymphomas expressing the ectopic anti-apoptotic protein $\mathrm{Bcl}-2$ (E $\mu-M y c$; Bcl2). E $\mu-M y c ; \operatorname{Trp}^{-/} 3^{-}$tumours showed a delayed regression followed by recurrence after cyclophosphamide (CTX) treatment. By contrast, E $\mu-M y c ; B c l 2$ tumours, which 
are resistant to apoptosis, showed no alteration in tumour size and hence appeared not to have responded to CTX. However, the prognosis of these mice was significantly better than the $\mathrm{E} \mu-M y c ; \operatorname{Trp}^{-/-}$mice because the cells in the $\mathrm{E} \mu-M y c ; B c l 2$ lymphomas became senescent, although the $\mathrm{E} \mu-M y c ; B c l 2$ lymphomas also eventually progressed. p53-dependent senescence could also be induced in $\mathrm{E} \mu-\mathrm{Nras}^{\mathrm{G}}{ }^{\mathrm{D}}$; Bcl2 lymphomas treated with chemotherapy ${ }^{22}$ and in $M y c$-driven lymphomas in response to Myc inactivation ${ }^{33}$. It was also shown that reactivation of endogenous p53 induces senescence in various p53-defective tumours, including $M y c$-driven lymphomas, Hras $^{\mathrm{G} 12 \mathrm{~V}}$-driven liver cancer, and sarcomas ${ }^{34-35,36}$. Although p53 has important roles both in apoptosis and senescence, these studies suggest that the p53-mediated senescence pathway can affect therapeutic outcome, particularly in an apoptosisdefective context. Some of the most relevant models of therapy-induced senescence in the in vivo setting are summarized in Table 1 , and reviewed in ${ }^{37-39}$.

\section{Senescence and intracellular pathways}

[Au; for clarity and flow, I think that Box 3 should be incorporated into the main text here (even though this will increase the length of the main text). Please ensure that the references are in the correct order when you make this change.]

\section{Mitochondrial metabolism}

Recent studies on senescence-associated metabolic features have provided insights into cancer biology and therapy. Specific metabolic programs in cells adapt to genetic changes or environmental cues. For example, tumour cells often exhibit a shift in glucose metabolism from mitochondrial oxidative phosphorylation to glycolysis even 
in the presence of oxygen (the Warburg effect) ${ }^{40}$ (FIG 1). Emerging evidence indicates that senescent cells also shift glucose metabolism, but in a way that is distinct from proliferating cancer cells $\mathrm{s}^{41-43}$.

Kaplon et al. ${ }^{41}$ recently showed that increased mitochondrial oxidative phosphorylation is critical for OIS induction. Pyruvate is at the node between glycolysis and the mitochondrial tricarboxylic acid (TCA) cycle. Pyruvate can be converted by the pyruvate dehydrogenase (PDH) complex to acetyl-CoA, which enters the TCA cycle (FIG. 1). Cells in culture expressing either oncogenic BRAFV600E or RAS-G12V that undergo OIS have increased PDH activity ${ }^{41}$. Downregulation of PDH activity abrogated BRAF-V600E-induced senescence, whereas activation of PDH was sufficient for senescence induction in normal cells, indicating a key role for PDH in OIS. A similar increase in mitochondrial metabolism, resulting in increased oxygen consumption, energy production, lipid catabolism and increased levels of reactive oxygen species (ROS), was found in TIS of $\mathrm{E} \mu-M y c ; B c l 2$ lymphomas ${ }^{43}$.

These studies highlight the stark contrast between senescent and proliferating tumour cells with respect to the pyruvate flux into the TCA cycle for efficient energy production (FIG. 1). Interestingly, activation of PDH in mutant BRAF-driven human melanoma cells not only blocks tumorigenesis but also sensitises them to the BRAF inhibitor PLX4720 (an analogue of vemurafenib), eliminating treatment-resistant cell populations ${ }^{41}$. This finding is encouraging, because although the ATP-competitive BRAF inhibitors, vemurafenib and dabrafenib have shown dramatic effects on BRAFV600E-driven melanomas in the clinic, this response is almost inevitably temporary ${ }^{44}$. Thus, developing combination therapies with BRAF inhibitors and drugs that activate $\mathrm{PDH}$ might be a promising strategy. $\mathrm{PDH}$ is negatively regulated through 
phosphorylation by pyruvate dehydrogenase kinase (PDK). PDK1, one of four known PDK isozymes, is down-regulated in BRAF-V600E-induced senescence, and RNA interference (RNAi) against PDK1 induces sensitivity of the BRAF-V600E expressing cells to vemurafenib ${ }^{41}$. Dichloroacetate, a small molecule inhibitor of PDK, has been used in humans for the treatment of lactic acidosis and inherited mitochondrial diseases for decades and dichloroacetate has already been implicated in cancer therapy ${ }^{45,46}$. In theory, this drug could be used to inhibit PDK, and hence activate $\mathrm{PDH}$, in melanoma, but would all melanomas respond to this therapy?

Interestingly, recent studies have identified two distinct sub-groups in human melanoma: a conventional glycolytic group and a group characterised by high rates of oxidative phosphorylation ${ }^{47,48}$. In the latter group, microphthalmia-associated transcription factor (MITF), the oncogenic melanocyte lineage-specification transcription factor, drives peroxisome-proliferator-activated receptor $\gamma$ coactivator $1 \alpha$

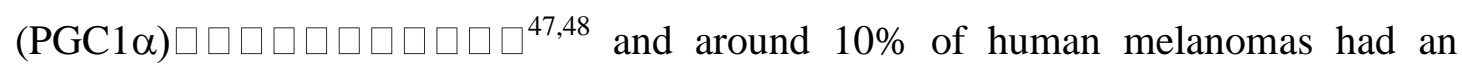
above average expression level of PGC1 $\alpha^{48}$. PGC1 $\alpha$ is a transcriptional coactivator and promotes mitochondrial respiration and, at the same time, induces many ROSdetoxifying enzymes ${ }^{49}$. Consistently, MITF and PGC1 $\alpha$-expressing melanoma cells are more resistant to oxidative stress. Importantly, no correlation was observed between PGC1 $\alpha$ expression and BRAF mutation status ${ }^{48}$. Thus it would be important to carefully validate the effect of PDK1 inhibition, with or without BRAF inhibitors, in the MITF and PGC1 $\alpha$-expressing subset of melanomas, which appear to be associated with a poor prognosis ${ }^{47,48}$.

Other metabolic pathways, such as the p53-mediated regulation of malic enzymes (MEs), have been implicated in the regulation of senescence. ME1 and ME2 expression is repressed by p53 and the MEs negatively regulate p53, thus depletion of 
the MEs results in p53 activation in a feed forward manner, thereby inducing senescence $^{50}$ (FIG. 1). However, it is possible that ME depletion-associated metabolic alterations also regulate senescence. MEs catalyse the oxidation of malic acid into pyruvate, using $\mathrm{NADP}+$ or $\mathrm{NAD}(\mathrm{P})+$ as cofactors. MEs are a major source of NADPH for the antioxidant system and lipid biosynthesis, and have also been implicated in increased glutamine consumption in tumours ${ }^{51}$. Thus MEs can potentially divert the TCA cycle intermediate, malic acid, to the synthesis of macromolecules to form building blocks. Jiang et al. ${ }^{50}$ showed that depletion of MEs induces senescence with the reduction of the NADPH levels. Consistently, these cells also show ROS induction and reduced lipid synthesis. In addition, although mitochondrial respiration was not tested in this context, a reduction in ME activity may facilitate TCA cycle flux by reducing the malate shunt, thereby reinforcing senescence-associated PDH activation (FIG. 1). Interestingly, ME2 depletion also induces differentiation in K562 erythroleukemia cells ${ }^{52}$. Although the role of the MEs in the context of OIS and TIS is still unclear ${ }^{50}$, these studies all seem to point to a senescence-associated metabolic shift toward maximal energy production at the expense of macromolecule synthesis and reducing power.

Consistent with this view, Quijano et al. ${ }^{53}$ showed that HRAS-G12V-induced senescent HDFs exhibit reduced lipid synthesis and increased fatty acid (FA) oxidation, leading to enhanced mitochondrial oxygen consumption. As Dörr et al. ${ }^{43}$ suggest, such a diversion into energy production would also be beneficial for supporting energy consuming activities in senescent cells, such as producing high levels of secretory proteins (as evident in a SASP) and autophagy (see below). Indeed, Quijano et al. ${ }^{53}$ showed that the inhibition of FA oxidation in OIS cells results in a reduced production of many SASP components. Unlike rapidly proliferating tumour 
cells, senescent cells would not need a continuous supply of macromolecules, such as lipids and nucleotides. Hence, it would be interesting to know whether the reduced capacity for building block production during senescence is a consequence or a cause of the phenotype. Finally, increased ROS levels due to hyperactive mitochondrial respiration as well as reduced levels of NADPH are also likely to contribute to senescence induction, further suggesting the robust involvement of a metabolic shift in senescence.

In contrast to these recent studies, suggesting the highly active state of mitochondrial respiration during OIS and TIS, early studies had associated senescence with mitochondrial dysfunction ${ }^{54}$, particularly in the ageing and replicative senescence contexts ${ }^{55,56}$. While it was recently shown that the metabolic profiles of OIS and replicative senescence HDFs are highly distinct ${ }^{53}$, inefficient mitochondrial energy production was also observed in the OIS model ${ }^{57}$. The reason for the apparent discrepancy is not clear. However, the dysfunctional mitochondria in HDFs that have undergone OIS or replicative senescence were accompanied by active mitochondrial biogenesis $^{55,57}$, with a concurrent up-regulation of $\mathrm{PGC} 1 \alpha^{57}$, which is a major regulator of mitochondrial biogenesis, thus increasing respiration, antioxidant defences, and FA oxidation ${ }^{49}$. Interestingly, they also noted increased mitochondrial mass at the perinuclear region ${ }^{57}$, where macroautophagy (refereed to as autophagy hereafter), the machinery for the bulk degradation of macromolecules and organelles, is often activated upon stress ${ }^{58-60}$. These data suggest the potential of active mitochondrial turnover in OIS cells. Interestingly, it was shown that inefficient "mitophagy" is associated with mitochondrial dysfunction in some tumour cells ${ }^{61}$. As we discuss below, autophagy can be induced during $\mathrm{OIS}^{59}$, and it can cause different effects on the senescence phenotype during OIS, depending on the level of its 
induction $^{62}$. In addition, the intensity of the oncogenic stress also has a profound effect on the dynamics of the proliferative and senescence responses ${ }^{63}$. Shifting the fine balance between mitochondrial biogenesis and recycling might, at least in part, explain the phenotypic heterogeneity.

\section{Autophagy}

Autophagy is a genetically regulated catabolic process where cytoplasmic components, including organelles, proteins and other macromolecules, are encircled by autophagic vesicles and are delivered to lysosomes for bulk degradation. Autophagy occurs both basally and in response to metabolic or other stresses ${ }^{64}$. Autophagy has key roles in ageing and cancer, but its links to senescence are just emerging 65 .

The role of autophagy in cancer is multifactorial and highly context dependent $^{66,67}$. Autophagy is recognised as a survival factor for tumour cells under metabolic and therapeutic stresses ${ }^{66,68}$. However, genetic evidence also suggests tumour suppressive roles for autophagy ${ }^{69-72}$.

Increased autophagic activity during senescence has been shown in different models ${ }^{4,73}$. SA-ß-gal is a lysosomal enzyme ${ }^{74}$ and is frequently expressed in senescent cells that have autophagic vesicles ${ }^{58,75}$, suggesting that autophagy might be an integrated part of the senescence program. Its functional role in senescence, however, appears to differ depending on the experimental system ${ }^{65}$. Autophagy is dynamically activated during OIS and facilitates the synthesis of interleukin 6 (IL-6) and IL-8, major components of the $\mathrm{SASP}^{58,59}$. A positive correlation between autophagy and senescence has also been observed in other models including OIS and TIS in culture, where the inhibition of autophagy often delays senescence induction ${ }^{76-82}$. Consistent 
with its cytoprotective function, blocking autophagy during senescence induction can also result in cell death ${ }^{83}$ or even the exacerbation of senescence ${ }^{62,84}$. One speculative explanation for this apparent discrepancy would be that, in addition to the role of autophagy on senescence effectors (for example, the SASP), its cytoprotective effect could divert the stress response towards senescence rather than cell death. Thus the outcome of autophagy deficiency in pro-senescence conditions may be senescence bypass if cellular fitness had already been secured, or otherwise a severe reduction of cellular fitness: thus autophagy might affect the 'quality' of senescence.

While data on the roles of autophagy in senescence in vivo are relatively limited, ${ }^{43,59}$ in their E $\mu-M y c ; B c l 2$ model, Dörr et al. recently showed that activated autophagy has a pro-survival role, protecting against proteotoxic stress, which is associated with an increased production of SASP components during TIS $^{43}$. Thus, in contrast to the OIS model in culture, autophagy inhibition results in the selective killing of tumours due to endoplasmic reticulum (ER) stress in this context.

Although the majority of studies have indicated that autophagy inhibition is beneficial for cancer patients (reviewed in ${ }^{85,86}$ ), it is not always the case. Recently, Rosenfeldt et al. ${ }^{87}$ have investigated the role of autophagy at different stages of $\operatorname{Kras}^{\mathrm{G} 12 \mathrm{D}}$-driven pancreatic tumorigenesis. These mice develop pancreatic intraepithelial neoplasias (PanINs), which express markers of senescence ${ }^{24}$, however these lesions can eventually progress to pancreatic ductal adenocarcinoma (PDA). In this model, autophagy deficient mice exhibit a marked but transient accumulation of early PanIN accompanied by increased rates of apoptotic cell death, and these lesions never progress to late stage PanINs or $\mathrm{PDA}^{87}$. Thus, autophagy seems to have a cytoprotective role in this setting. However, the same study showed that when $\operatorname{Kras}^{\mathrm{G} 12 \mathrm{D}}$-driven PDA development is accompanied by loss of Trp53 (and is thus 
senescence defective), autophagy deficiency instead accelerated tumour progression. Autophagy deficient cell lines derived from these tumours had higher levels of intracellular glucose, glycolytic intermediates and components of the pentose phosphate pathway whereas the TCA cycle was unaffected, suggesting that the autophagy defect in Trp53-null PDA provides metabolic advantages for proliferation, reminiscent of the Warburg effect.

Interestingly, the effect of autophagy inhibition in $\mathrm{Kras}^{\mathrm{G} 12 \mathrm{~V}}$-driven lung tumorigenesis (another OIS model ${ }^{24}$ ) is distinct from the pancreas model. In the lung model, regardless of p53 status, autophagy deficiency diverts adenoma or adenocarcinoma into oncocytoma, a rare and predominantly benign tumour characterised by the accumulation of respiration-defective mitochondria ${ }^{88}$. Thus, in contrast to the pancreas model, autophagy deficiency (by autophagy related 7 (Atg7) knockout) suppresses $\operatorname{Kras}^{\mathrm{G} 12 \mathrm{D}}$-driven lung cancer development even in the absence of Trp53; presumably this is partially due to mitochondrial dysfunction. This is consistent with the previous study from the same group that found that autophagy inhibition in nutrient starved conditions in cells that are 'addicted' to autophagy (particularly cells expressing oncogenic RAS) can result in cell death accompanied by the accumulation of dysfunctional mitochondria ${ }^{61}$.

Although the precise role of autophagy in these OIS models is still unclear, these studies highlight the complex link between autophagy and mitochondrial respiration. Autophagy also appears to be required for the increase in oxygen consumption during RAS-induced senescence in MEFs, and TIS cells with hyperactive mitochondrial respiration die on autophagy inhibition ${ }^{43}$. Thus to reconcile the apparently distinct roles for autophagy in tumorigenesis between the different tissues, it is tempting to speculate that autophagy might somehow regulate the balance 
between glycolysis and mitochondrial respiration, favouring the latter and, depending on the underlying metabolic stress, cells would develop either a glycolytic state or metabolic crisis $^{61}$. It is also possible that the activated mitochondrial respiration reported in senescence models is, at least in part, dependent on intact autophagy.

\section{Senescence and the extracellular environment}

\section{Senescence-associated secretory phenotype (SASP)}

The tumour suppressor function of senescence has been largely attributed to the lack of proliferative capacity of senescent cells. However, increasing evidence, particularly from sophisticated mouse models, indicates that senescent cells actively communicate with neighbouring cells and the extracellular matrix through a plethora of secretory factors, including extracellular proteases, cytokines and chemokines, and growth factors. Such studies have revealed the functionality as well as the dynamic longerterm fate of senescent cells in vivo ${ }^{89,90}$. The SASP (also called the senescencemessaging secretome, SMS) adds enormous complexity to the implications of senescence in cancer. The nature of the SASP and its targets, and the overall downstream outcomes, vary considerably depending on the cellular context, type of stress, and type of model used (FIG. 2).

Various factors regulate SASP, both at the transcriptional and posttranscriptional levels, contributing to the diverse outputs of senescence ${ }^{58,59,91-96}$. Importantly, SASP factors seem to form a hierarchical network, in which the signal is transduced and amplified. For example, senescence was recently shown to be closely associated with inflammasomes, which play a central role in inflammation ${ }^{93,97}$. This concept predicts the existence of master regulator(s) of the SASP. Currently, proinflammatory cytokines, including IL-1 and IL-6, and their regulators have been 
proposed as major upstream regulators of SASP, and inhibition of either of them results in the collapse of the cytokine network in culture ${ }^{91,93,98,99}$.

Some SASP components, for example matrix metalloproteinase 3 (MMP3) and plasminogen activator inhibitor 1 (PAI1), have long been used as markers of senescence ${ }^{100,101}$. Although PAI1 has since been shown to be a positive effector of senescence $^{102}$, some soluble factors, such as MMP3, were known to promote tumorigenesis in neighbouring pre-malignant epithelial cells through paracrine effects $^{103,104}$. Later studies reinforced the importance of the paracrine activities of the SASP as IL-6 and IL-8 secreted from senescent cells promote tumorigenesis in premalignant, but not normal cells. However, these same factors can facilitate senescence in a cell-autonomous fashion, thus amplifying the phenotype ${ }^{91,92,105}$, and paracrine pro-senescence factors, such as insulin-like growth factor binding protein 7 (IGFBP7), IL-1 and transforming growth factor- $\beta$ (TGFß), were also identified ${ }^{6,93,106,107}$, although the role of IGFBP7 as a paracrine senescence mediator in melanocytes is a matter of debate $^{108,109}$. Thus it is conceivable that, in addition to its cell-autonomous role, senescence could also confer a tumour suppressive microenvironment during the early stages of tumorigenesis. However, this might be at the risk of a sudden change of senescence to a tumorigenic factor with the appearance of 'senescence-escapers' (or de novo pre-malignant cells). These concerns would also be relevant in attempting to use the induction of senescence as a cancer therapeutic goal, where the SASP might drive the proliferation of surviving tumour cells ${ }^{110}$.

\section{Clearance of senescent cells through an immune response}

Increasing evidence indicates that senescent cells can be eliminated through a SASPprovoked immune response, which involves both innate and adaptive immunity ${ }^{111,12}$ 
(FIG. 2). The involvement of an immune response also extends the anti-tumour role of the SASP toward established transformed cells in some tumour models.

The first evidence for the clearance of senescent cells through an immune response came from a mouse liver carcinoma model where endogenous p53 could be restored by turning off Trp53-targeted RNAi after tumours had been established ${ }^{34}$. Re-expression of endogenous p53 induced senescence and the elimination of the senescent tumour cells was mediated by innate immune cells, including macrophages, neutrophils and natural killer (NK) cells, albeit in mice lacking an adaptive immune system. More recently, using a similar system Iannello et al. ${ }^{113}$ focused on the role of NK cells and described how the NK cells mediate elimination of senescent tumour

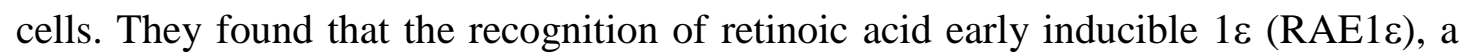
ligand expressed by tumour cells, by NKG2D (also known as KLRK1), an activating receptor expressed in all NK cells, is critical for the elimination of senescent tumors. NKG2D has been implicated in the detection and elimination of transformed and virus-infected cells ${ }^{114}$. These ligands are induced in response to stress pathways that are associated with tumorigenesis, and indeed RAE1ع [Au; any particular isoform?] in liver tumour cells was highly expressed before and after $\mathrm{p} 53$ restoration ${ }^{113}$. This raises an interesting possibility that senescence induction in just a subset of tumour cells would allow NK cells to kill not only senescent but also non-senescent tumour cells if NK cells could be recruited to the lesion (FIG. 2). The immunosuppressive nature of the tumour microenvironment is known to limit the capacity of immune cells to interact with and kill tumour cells ${ }^{115}$. Thus, it would be interesting to know whether, in addition to the increase in the level of chemoattractants, senescence induction in tumour cells also causes a reduction in immunosuppressive factors produced by tumour cells and stromal cells. 
Although the innate immune response is sufficient for the clearance of p53 reactivation-induced senescent cells from the liver, the adaptive immune system was also shown to have an important role during tumour senescence in some mouse $\operatorname{models}^{116}$. For example, the depletion of MYC in a mouse subcutaneous transplantation model of $\mathrm{T}$ cell acute lymphoblastic lymphoma (T-ALL), which carries tetracycline regulated $M Y C$ transgene, results in sustained tumour regression associated with senescence and apoptosis, where an adaptive immune response (involving $\mathrm{CD}^{+} \mathrm{T}$ cells in particular) was required for senescence induction and sustained tumour regression, but not for apoptosis induction ${ }^{116}$. Although altered levels of several cytokines were evident on MYC-depletion, this study did not examine how the senescent cells were cleared.

A recent study conducted by Kang et al. ${ }^{112}$ addressed this question in great detail in the mouse liver OIS model, where the clearance of senescent cells also requires $\mathrm{CD}^{+} \mathrm{T}$ helper $\left(\mathrm{T}_{\mathrm{H}}\right)$ cells. They showed that stably transduced oncogenic $\operatorname{Nras}^{\mathrm{G} 12 \mathrm{~V}}$ drives the SASP in hepatocytes in mice and that these senescent cells are surrounded by various immune cells and are cleared predominantly by infiltrating monocytes and macrophages ${ }^{112}$. The elimination of senescent cells by innate immune cells requires the $\mathrm{CD} 4^{+} \mathrm{T}_{\mathrm{H}} 1$ cell response. Indeed, in livers bearing $\mathrm{Nras}^{\mathrm{G} 12 \mathrm{~V}}$-induced senescent hepatocytes, $\mathrm{CD} 4^{+} \mathrm{T}$ cells that recognize the new epitope formed by the G12V mutation in Nras, were identified. Remarkably, Nras-G12V-specific CD4 ${ }^{+} \mathrm{T}$ cells were absent when $\operatorname{Nras}^{\mathrm{G} 12 \mathrm{~V}}$ was transduced into the livers of senescencedeficient mice; or a double mutant of $\mathrm{Nras}^{\mathrm{G} 12 \mathrm{~V} / \mathrm{D} 38 \mathrm{~A}}$ was used, which cannot induce senescence. Furthermore, similar to the T-ALL senescence model, $\mathrm{CD} 4^{+} \mathrm{T}$ cells are required for sustained tumour regression. Thus this tumour associated antigen (TAA)- 
driven 'senescence surveillance' is an essential part of the tumour suppressor function of senescence.

Which aspect of senescence contributes to the immune clearance of premalignant cells in vivo? Although this is not clear, it is conceivable that a senescence specific cocktail of immunomodulatory factors might be a key determinant. SV40 T-antigen (Tag) driven spontaneous hepatomas evade TAA-driven immune surveillance because of T-cell tolerance ${ }^{117}$. Although Tag is highly immunogenic, it binds both p53 and RB, thus efficiently blocking the senescence response. Interestingly, mice with Tag-driven hepatoma have high levels of TGF-ß1, which effectively suppresses T cell function but low levels of circulating interferon $\gamma$ $(\mathrm{IFN}-\gamma)^{117}$. Such a strong dominance of TGF-ß1 function over IFN- $\gamma$ might contribute to the T-cell tolerance of Tag in these mice.

How general is 'senescence surveillance'? Interestingly, in oncogenic $\operatorname{Kras}^{\mathrm{G} 12 \mathrm{D}}$-driven PanIN Kras-G12D seems to be non-immunogenic ${ }^{115}$. The reason for the contrast between the liver and pancreas models is unclear. However, it is noteworthy that in contrast to Kras-G12D activation during embryogenesis in the PanIN model, Nras ${ }^{\mathrm{G} 12 \mathrm{~V}}$ was introduced directly into adult somatic cells in the liver. Thus the Kras-G12D epitope might be seen as a self-antigen and be subject to peripheral immune tolerance in the PanIN model. Interestingly, it was shown that pancreatic acinar cells, in which Kras-G12V is activated during adulthood, exhibit a low frequency of PanIN development and resistance to transformation, at least in part due to $\mathrm{OIS}^{118}$. It is conceivable that tumour susceptibility is different depending on the stage(s) of differentiation at which the cells are exposed to oncogenic stress. However, an alternative, highly speculative, explanation may be that adult onset of Kras-G12V activation in the pancreas may provoke senescence immune surveillance. The same 
study also showed that episodic pancreatitis induced by caerulein, a decapeptide analog of the gastrointestinal peptide hormone cholecystokinin, inhibited the OIS program, thus facilitating malignant transformation in this model ${ }^{118}$. Further detailed characterisation of the immune response induced by either OIS or tissue damage in these different models will be necessary. Of note, it seems that senescence arrest can be tumour suppressive without any obvious involvement from senescence surveillance: human melanocytic naevi (an OIS model), remain non-proliferative for decades without transformation into malignant melanoma ${ }^{25}$. These observations collectively underscore the diversity of the communication between senescent cells (or senescent cells-to-be) and immune effector cells in different scenarios.

\section{Senescence in the stroma}

The paracrine activity of senescent fibroblasts on epithelial cells was originally shown primarily in in vitro co-culture or xenograft models, where fibroblasts induced to senesce in culture promoted the proliferation and invasiveness of their neighbouring (pre)malignant epithelial cells ${ }^{91,103-105,119}$. However, Scott Lowe's group pioneered a mouse model to show stromal senescence in the in vivo setting ${ }^{120}$ (FIG. 3). During liver injury, quiescent hepatic stellate cells (HSCs) are activated into proliferative and fibrogenic myofibroblasts, which contribute to liver fibrosis ${ }^{121}$. Using a wellestablished mouse model of liver fibrosis induced by chronic treatment with the liverdamaging agent carbon tetrachloride $\left(\mathrm{CCl}_{4}\right)$, they showed that activated $\mathrm{HSCs}$ become senescent, exhibiting a typical SASP, including the expression of extracellular matrix degrading enzymes and proinflammatory cytokines. The SASP also attracts innate immune cells that in turn eliminate the senescent HSCs and facilitate the resolution of fibrosis $^{94,120}$. Therefore, timely activation of HSCs and elimination of senescent 
activated HSCs are critical for tissue integrity in this model. Although in this study it was not clear how activated HSCs become senescent, a separate study showed that the matricellular protein CCN1 (also known as CYR61), which is secreted from damaged hepatocytes, can trigger senescence in activated $\mathrm{HSCs}^{122}$ (FIG. 3). CCN1 can also promote myofibroblast senescence, which is associated with the expression of antifibrotic genes in the skin, thus CCN1 mediated stromal senescence might be involved in wound healing ${ }^{123}$.

These data indicate that senescence in the context of some forms of tissue damage involves the spatio-temporal control of secreted protein levels as well as the content of SASP, which in turn modulates the senescence phenotype and dynamically regulates the surrounding tissue integrity; eventually contributing to the resolution of the lesion. Such a dynamic senescence process also seems to affect local tumorigenesis. In addition to fibrosis, senescent activated HSCs limit hepatocellular carcinoma (HCC) development in the presence of the chemical carcinogen diethyl nitrosamine (DEN), at least in part through activating a 'classical' tumour-suppressive M1 phenotype of macrophages, which provides an anti-tumour microenvironment and eventually eliminates senescent HSCs (FIG. 3). Interestingly, senescence in activated HSCs and the subsequent SASP-mediated activation of M1-macrophages are largely dependent on p53: Trp53-deficient HSCs continue to proliferate, stimulate the tumour-promoting M2 macrophages, and increase both cirrhosis and HCC development. Thus, p53 has a non-cell autonomous tumour suppressive role ${ }^{94}$. The non-cell autonomous tumour suppressive role for p53 was also proposed by an earlier study, where p53 was shown to buffer the expression of tumorigenic SASP components in senescent cells, thus restraining the tumorigenic microenvironment ${ }^{105}$. 
These studies exemplify the anti-tumour effects of stromal cell senescence and the accompanying SASP. However, another recent study suggests that the SASP of senescent HSCs can also promote tumorigenesis. Dietary or genetic obesity in mice can affect gut microbiota, and increased concentrations of the gut bacterial metabolite deoxycholic acid (DCA), which occurs during obesity, induces a SASP in HSCs, via enterohepatic circulation. In the presence of the chemical carcinogen DMBA, DCA promotes $\mathrm{HCC}$ development in the obese mice ${ }^{97}$. Thus, in contrast to the $\mathrm{CCl}_{4^{-}}$ induced liver damage model, DCA-induced senescent HSCs in the obesity model seem to be tumorigenic. The reason behind the apparently opposing effects of senescent HSCs on HCC development is not known. The long-term fate of senescent HSCs and the nature of the immune response triggered by the SASP were not examined in the obesity model. However, it was noted that, unlike the $\mathrm{CCl}_{4}$ treated mice, the obese mice did not develop fibrosis in the liver, suggesting that the nature of the tumour microenvironment may be substantially different between these two models. The authors fed DCA to non-obese mice and DMBA failed to increase HCC development. Thus some other obesity-related pro-tumorigenic factors may cooperate with senescent HSCs to facilitate the pro-tumorigenic microenvironment. To better understand the switch in the impact of SASP on tumorigenesis, it would be important to comprehensively compare the SASP components of senescent HSCs between the two models.

Stromal senescence was demonstrated more robustly using p16-reporter mice $^{124}$. With a number of different cancer models in the p16-reporter mouse, including transgenic autochthonous, transplanted and spontaneous tumours, the authors showed that early neoplastic events induce stromal senescence often much earlier than the tumours became palpable. Although further senescence validation 
using additional markers is necessary, these remarkably similar findings across different tumour models suggest that stromal senescence activated by neoplastic events is generally tumorigenic. However, it is not entirely clear whether tumours develop due to, or despite of, the SASP of the stromal cells in these models.

\section{Cytokine-mediated senescence}

Recent studies have shown that some SASP components not only reinforce the senescence response in cells that are already primed for senescence, but also induce senescence in normal neighbouring cells in a paracrine fashion ${ }^{93,106}$. However, these senescence-inducing soluble factors have different sources. For example, the chemokine GRO1 (also known as CXCL1), which is secreted from transformed cells, can induce senescence in stromal fibroblasts ${ }^{125}$. In an E $\mu-M y c$ lymphoma model, p53induced apoptosis of lymphoma cells led to the activation of macrophages to secrete TGFß1, which in turn induced senescence in lymphoma cells ${ }^{36}$. Thus tumour apoptosis can locally provoke cytokine-mediated senescence in this model. Note this study also reinforces the non-cell autonomous tumour suppressor role of p53, as mentioned above. Another example involves adaptive immunity and $\mathrm{T}_{\mathrm{H}} 1 \mathrm{~T}$ cell cytokines that can directly drive cancer cells into senescence ${ }^{126}$. Using the classical SV40 Tag-induced carcinogenesis model of pancreatic cancer, where Tag is expressed in pancreatic islets under the control of the rat insulin promoter (RIP-Tag2 mice ${ }^{127}$ ), Tag-activated $\mathrm{CD}^{+} \mathrm{T}_{\mathrm{H}} 1$ cells expressing a $\mathrm{T}$ cell receptor specific for the Tag peptide (Tag- $\mathrm{T}_{\mathrm{H}} 1$ cells) have a tumour suppressive role, which requires that the target tumour cells respond to both the IFN $\gamma$ and tumour necrosis factor (TNF) signals produced by the Tag- $\mathrm{T}_{\mathrm{H}} 1$ cells ${ }^{128}$. Although Tag binds both RB and $\mathrm{p} 53$, it attenuates, rather than fully inactivates, their activity, thus their residual activities allow the RIP- 
Tag2 mice to exhibit progressive tumorigenesis with only a small portion of islets developing invasive pancreatic cancer ${ }^{127,129}$. Treatment of mice with Tag- $\mathrm{T}_{\mathrm{H}} 1$ cells in this model induces a cell proliferation arrest in the tumors and improves the survival of these mice ${ }^{128}$. The TAA-expressing islets treated with Tag- $\mathrm{T}_{\mathrm{H}} 1$ cells are functional (i.e. they produce insulin), thus appeared to be in a state of tumour dormancy ${ }^{128}$. Although the relationship between tumour dormancy and senescence may be a matter of debate, the same group later showed that these cells were arrested due to senescence ${ }^{126}$. The senescence phenotype is dependent on the p16-RB pathway, thus unlike the aforementioned Tag-driven hepatoma model, the residual RB activity seems to be sufficient for senescence induction in this model ${ }^{129}$. Importantly, if the tumour cells fail to respond to either IFN or TNF, Tag- $\mathrm{T}_{\mathrm{H}} 1$ cells do not just fail to suppress tumour growth, instead they promote tumour growth in RIP-Tag2 mice ${ }^{128}$.

A link between tumour dormancy and the immune response has been observed in different experimental tumour models ${ }^{130}$. For example, studies in a spontaneous mouse melanoma model suggest that dormancy in micrometastatic lesions is mediated by immunosurveillance ${ }^{131,132}$. In this model, tumour cells disseminate early during tumorigenesis, and these cells remain dormant for prolonged periods of time. Interestingly, cytostatic $\mathrm{CD}^{+} \mathrm{T}$ cells suppress the expansion of the disseminated tumour cells ${ }^{132}$. Precisely how $\mathrm{CD}^{+} \mathrm{T}$ cells induce tumour dormancy is not shown in this model, however, $\mathrm{CD}^{+} \mathrm{T}$ cells also secrete cytostatic cytokines, including IFN $\gamma$ and TNF. Thus, the combination of IFN $\gamma$ and TNF induced-senescence in particular, might widely contribute to tumour control. Consistently, Braumüller et al. ${ }^{126}$ confirmed that IFN $\gamma$ and TNF induce senescence in a number of different cancers both in vitro and in vivo. 
Chronic inflammation is generally a risk factor for tumorigenesis ${ }^{133}$, however, psoriasis, an immune-mediated cutaneous chronic inflammatory disease is associated with a reduced risk for cancer ${ }^{134}$. It is suspected that this might be because of senescence $^{135}$. The development of psoriatic plaques is accompanied by activation of $\mathrm{CD}^{+} \mathrm{T}$ cells and other immune cells, which provide a plethora of cytokines and chemokines, including TNF and IFN $\gamma^{136}$. Here also a TNF and IFN $\gamma$ cocktail might provide a dominant tumour suppressor activity through cytokine-induced senescence.

\section{Concluding remarks}

Recent advances in our understanding of senescence biology have substantially extended the known pathophysiological roles of senescence. These studies suggest that senescence is a more widespread phenomenon than was previously thought (BOX 1). The functional association of senescence with other regulatory phenotypes, such as mitochondrial metabolism and the immune response, requires further study. For example, metabolic intermediates of the TCA cycle affect epigenetic-mediated control of gene expression through regulating the modification of histones and DNA ${ }^{137,138}$. These pathways might well be altered in senescent tumour cells and stromal cells. Although in this review we have treated carbohydrate metabolism as a cellautonomous effector, it is conceivable that metabolic intermediates from different tissue compartments can transduce signals and nutrients to each other. Indeed, it has been proposed that the lactate extruded from glycolytic tumour cells may be taken up by tumour cells (and potentially senescent cells) undergoing oxidative phosphorylation to fuel the TCA cycle ${ }^{139}$.

Senescent cells are by definition irreversibly arrested, however, in the senescence immunosurveillance model, senescence per se may not be a very efficient 
tumour suppressor, at least in some tumour models. The analogy between senescence and dormancy in some tumours also implies that the in vivo senescence-like arrest may be unstable, because dormant tumour cells can regrow after long periods of time ${ }^{130,140}$. It is possible that a rare stochastic event of senescence escape occurs, in some cases facilitated by SASP, during the course of tumorigenesis. Much like dormant cells, senescent cells are resistant to apoptosis or mitotic catastrophe upon chemotherapy, thus if senescent tumour cells are not eliminated, the sustained expression of pro-tumorigenic cytokines can lead to a worse outcome ${ }^{110}$. It is also possible that the heterogeneity and complexity of the senescence phenotype may confer a spectrum of senescence intensity or quality ${ }^{4}$, thus in some contexts or tissues, coupling a stable cell cycle arrest with the eventual clearance of the senescent cells is necessary to limit tumorigenesis.

Such a diverse senescence response is also relevant when considering senescence as a therapeutic option. For example, the outcome of endogenous p53 reactivation can be senescence and/or cell death depending on the tumour type $\mathrm{e}^{35,141,142}$. Even in the same tissue, the same triggers can either suppress (by inducing senescence) or promote tumorigenesis, as in the case for cytokine-induced senescence ${ }^{128,143}$. The partial induction of senescence in tumours could eradicate or exacerbate the tumours ${ }^{110,113}$. Either in an immune-mediated or non-immune manner, mechanisms for the selective elimination of senescent cells need to be explored further. Researchers have just started to accumulate examples of the high-order communication of senescence with the microenvironment in several in vivo models, but our current knowledge suggests that carefully individualised medical indications need to be considered when targeting senescence effectors for cancer therapy. A more detailed understanding of the integrity of the SASP in terms of both the expression of 
the components and also its functional networks would help us to exploit senescence for optimal tumour suppression.

\section{Acknowledgements}

We thank C. Frezza and D.T. Fearon for their thoughtful discussions, and M Hoare, Masako Narita, and other members of Narita group for critical reading and discussions. This work was supported by the University of Cambridge, Cancer Research UK, Hutchison Whampoa, and the Human Frontier Science Program (M.N.). 


\section{Box 1: Consequences of cellular senescence: aging and development}

Besides its role in tumour suppression and tumorigenesis cellular senescence has been linked to other processes, including aging and, most recently, mammalian embryonic development.

Aging: The functional role of cellular senescence in organismal ageing is not entirely clear. On the one hand, accumulation of several senescence markers in aged individuals has been reported in various tissues ${ }^{56}$. On the other hand, the inverse correlation between the replicative lifespan of cells (such as skin fibroblasts) in culture and donor age appears to be controversial ${ }^{144}$. Thus ageing affects senescence, but how about the other way around? The compelling evidence for the functional relevance of senescence in ageing was shown in mice where age-dependent accumulation of p16-positive cells in tissue stem cell or progenitor cell compartments contributes to tissue degeneration, and p16 depletion attenuates some age-dependent phenotypes ${ }^{145-147}$. Interestingly, selective killing of those p16-expressing cells in a premature ageing mouse model also delays ageing phenotypes ${ }^{148}$. Importantly, unlike p16-ablation, elimination of p16-expressing cells does not facilitate tumorigenesis in this model. It is possible that the efficient turnover of stem or progenitor cells facilitates tissue regenerative capacity. It is also possible that ablation of the senescent cells attenuates the local and perhaps systemic effects of the secretory associated senescent phenotype (SASP), which might contribute to various aspects of ageing.

Embryogenesis: Recently, two studies have uncovered a physiological function for senescence during mammalian embryonic development. Embryonic cell senescence is p21, but not p53, dependent, is accompanied by the SASP, and these cells are 
eliminated by macrophages ${ }^{149,150}$. Thus, similar to apoptosis, senescence also contributes to embryonic patterning. Another study identified senescence in normal placenta, where cell fusion, mediated by the highly fusogenic glycoprotein endogenous retrovirus group W member 1 (ERVWE1), provides the maternal-fetal interface $^{151}$. They showed that ERVWE1-mediated cell fusion induces senescence. Thus, senescence, which was long thought to be purely a stress response, also appears to be physiologically programmed.

\section{Box 2: PTEN loss-induced cellular senescence (PICS)}

Among senescence induced by loss of tumour suppressors, PTEN loss-induced cellular senescence (PICS) in prostatic intraepithelial neoplasia (PIN) has been extensively studied by Pandolfi's group ${ }^{38,152}$. Pten heterozygosity promotes tumour initiation and proliferation in the prostate, but paradoxically, the complete loss of Pten can oppose prostate tumorigenesis by triggering a powerful p53-dependent cellular senescence response ${ }^{38}$. Consistently, the majority of prostate cancer patients at presentation have lost one allele of PTEN while maintaining intact TP53; whereas TP53 mutations and the complete loss of PTEN are both associated with advanced stage prostate cancer ${ }^{153,154}$. Alimonti et al. demonstrated a proof of principle that pharmacological inhibition of PTEN in prostate cancer that is wild type for TP53, results in the inhibition of tumorigenesis with senescence induction in a human xenograft model $^{152}$. Since PTEN is a negative regulator of the PI3K-mTOR pathway, which is also often considered to be a target for cancer therapy, targeting PTEN may sound counter-intuitive. Interestingly, TP53 up-regulation upon PTEN inhibition appears to be dependent on mTOR activity, which increases TP53 translation $^{152}$. Thus in some specific cases, such as tumours in which one allele of PTEN has become 
inactive, PTEN inhibitors might be an option for cancer therapy. Of note, unlike OIS, PICS occurs in the absence of transient hyper-proliferation and a DNA damage response (DDR) ${ }^{152}$. As we discuss in the main text, pro-senescence treatment may provoke a tumorigenic microenvironment through the SASP in some contexts. The SASP, however, has been shown to be mainly regulated through persistent $\mathrm{DDR}^{96}$. Thus PICS might provide a unique opportunity, where pro-senescence therapy may be separable from the potential side effects derived from the SASP. It would be interesting to know whether PICS, which has been shown not to be associated with DDR, is accompanied by some type of SASP and, if so, how it affects the tumour microenvironment. 


\section{Figure 1. Glucose metabolism in OIS, TIS and cancer.}

Active mitochondrial metabolism has been shown in oncogene-induced senescence $(\mathrm{OIS})^{41,53}$ and therapy-induced senescence (TIS) ${ }^{43}$. In the latter, active glucose uptake is maintained. The activity of pyruvate dehydrogenase (PDH) is positively and negatively regulated by pyruvate dehydrogenase phosphatases (PDP) and pyruvate dehydrogenase kinases (PDK), respectively. PDH is activated in senescent cells, induced by oncogenic BRAF or RAS, and PDH activation is accompanied by the induction of PDP2 and the suppression of PDK1 in BRAF-driven, but not in RASdriven, senescence ${ }^{41}$. Down-regulation of malic enzymes (MEs) in both fibroblasts and some tumour cell lines induces senescence ${ }^{50}$. In mammals there are three ME isoforms: the cytosolic, NADP+-dependent, ME1 (not shown in the figure); mitochondrial, NAD+ and NADP+ dependent, ME2; and mitochondrial, NADP+dependent, ME3. Although ME2 has been suggested to favour NAD+ over NADP+ as a cofactor, thus generating primarily NADH, it was shown that depletion of ME2 in cells in culture induces senescence with the reduction of the NADPH, but not NADH, levels $^{50}$. The relevance of the levels of MEs in OIS models is not clear. Note this figure represents a collective view derived from recent studies using different models, thus should not be generalised. Ac-CoA, acetyl-CoA; FA, fatty acid; PPP, pentose phosphate pathway; TCA, tricarboxylic acid cycle.

Figure 2. The senescence associated secretory phenotype (SASP) and its implication for the tumour microenvironment.

a. General view: senescent cells actively communicate with their microenvironment through a plethora of secretory factors as part of the SASP. These factors can reinforce the establishment of senescence in their cell of origin in an autocrine manner. 
They can also trigger the establishment of senescence in neighbouring cells in a paracrine manner. These signals lead to further secretion and hence to an amplification of the SASP. Senescence in this manner also modulates the extracellular matrix, enhances the transformation of predisposed cells and reinforces tumour growth. In addition, the SASP activates the immune system to eliminate senescent cells, which may remove potentially tumorigenic factors from the microenvironment. b. Senescence induction in tumours; three scenarios have been suggested. Immune cells, such as neutrophils, macrophages and natural killer (NK) cells recruited to the tumour lesion by the SASP eliminate the senescent cells ${ }^{34}$ and possibly non-senescent tumour cells ${ }^{113}$. However, sustained senescent cells can also facilitate tumour development $^{110}$.

c. In the case of Nras-G12V expressing hepatocytes their oncogene-induced senescence SASP leads to their clearance, predominantly by the infiltrating monocytes and the resulting macrophages ${ }^{112}$. The elimination of these senescent cells by the innate immune cells requires the $\mathrm{CD} 4^{+} \mathrm{T}_{\mathrm{H}} 1$ cell response. The $\mathrm{CD} 4^{+} \mathrm{T}$ cell activation depends on the presence of professional antigen presenting cells (APCs). Whether or not this occurs in other tissues, such as the pancreas, remains to be elucidated.

\section{Figure 3. Senescence in the stroma of the liver}

During liver injury quiescent hepatic stellate cells (HSCs) become activated. The HSCs become proliferative and fibrogenic myofibroblasts, which contribute to liver fibrosis by producing the extracellular matrix (ECM). Chronic liver damage (for example with exposure to $\mathrm{CCl}_{4}$ ) causes the activated $\mathrm{HSCs}$ to become senescent in a p53-dependent manner, exhibiting the typical SASP, including ECM degrading 
enzymes and proinflammatory cytokines. The senescent activated HSCs thus limit excessive fibrosis (cirrhosis) and activate senescence surveillance through, for example, natural killer (NK) cells and anti-tumour M1 macrophages, and thereby limit the development of hepatocellular carcinoma (HCC $)^{94,120}$ (a). By contrast, in an obesity model, increased levels of the gut bacterial metabolite deoxycholic acid (DCA) provoked HSC senescence and the SASP promoted HCC development ${ }^{97}$ (b). The matricellular protein CCN1, which is secreted from damaged hepatocytes, can also trigger senescence in activated HSCs, thereby limiting fibrosis ${ }^{122}$.

\section{Glossary}

\section{Enterohepatic circulation}

The circulation between liver and intestine. Bile acids and other substances excreted from the liver are reabsorbed by the enterocytes into the hepatic portal vein.

\section{Inflammasome}

Cytosolic multiprotein innate immune complexes that assemble in response to a variety of stimuli, activating the pro-inflammatory serine protease caspase 1. Inflammasomes initiate innate immune responses by cleaving the inflammatory cytokines, pro-IL-1ß and pro-IL18, leading to their activation and secretion.

\section{Pancreatic intraepithelial neoplasias (PanIN)}

Induced by an activating mutation in KRAS and represent the most common precursor lesion of pancreatic ductal adenocarcinoma (PDA).

\section{NK cells}


A subgroup of white blood cells, implicated in the killing of tumour or virus infected cells. They are generally considered as the cytotoxic lymphocytes of innate immunity due to their lack of antigen-specific cell surface receptors, but they can also exert antibody-dependent cell cytotoxicity.

\section{T helper $1\left(\mathbf{T}_{\mathbf{H}} \mathbf{1}\right)$ cells}

A subset of $\mathrm{CD}^{+} \mathrm{T}$ helper cells that secrete cytokines, including IFN $\gamma$ and TNF. They are canonically associated with the stimulation of $\mathrm{CD}^{+}$cytotoxic $\mathrm{T}$ lymphocytes and of macrophages as well as anti-tumour immunity.

\section{macrophages}

Generally speaking, M1 polarised macrophages inhibit cell proliferation and cause tissue damage whereas M2 polarised macrophages promot cell proliferation and tissue repair. The $\mathrm{M} 1$ and $\mathrm{M} 2$ macrophages promote the $\mathrm{T}_{\mathrm{H}} 1$ and $\mathrm{T}_{\mathrm{H}} 2$ responses, respectively.

\section{Cytokines}

A broad class of small proteins that comprise chemokines, interferons, interleukins, lymphokines and tumour necrosis factors that are produced by a variety of cells.

\section{Warburg effect}

A shift in glucose metabolism from mitochondrial oxidative phosphorylation to glycolysis in the presence of ample oxygen.

\section{NADPH}


The reduced form of NADP+ and a source of reducing equivalents. Major sources of NADPH include the pentose phosphate pathway, NADP-dependent malic enzymes and NADP-dependent isocitrate dehydrogenase. 


\section{Author biographies}

Pedro Perez-Mancera is a senior scientist in Masashi Narita's laboratory at the CRUK Cambridge Institute (UK). He completed his $\mathrm{PhD}$ on the identification of molecular mechanisms involved in the development of myxoid liposarcoma at the University of Salamanca (Spain). Pedro developed his postdoctoral training in David Tuveson's laboratory at the Abramson Family Cancer Research Institute (Philadelphia, USA), and at the CRUK Cambridge Institute, where he unveiled novel molecular pathways involved in pancreas cancer progression using forward genetic screens. $\mathrm{He}$ is currently focused on exploring new pathways involved in induction of senescence in human neoplasms.

Andrew Young studied Biochemistry at the University of Wales, Swansea. He went on to undertake a $\mathrm{PhD}$ on autophagy at Cancer Research UK's, London Research Institute under Dr Sharon Tooze. He joined the Narita Lab in 2006, where he works on the association of autophagy and senescence.

Masashi Narita is a group leader in Cancer Research UK Cambridge Institute, University of Cambridge since 2006, studying effector mechanisms of cellular senescence. He obtained his MD in Osaka University School of Medicine (Japan) in 1992. After 4 years training as a surgeon, he started his $\mathrm{PhD}$ studies on apoptosis at Osaka University. In 2000, he finished his $\mathrm{PhD}$ and joined Scott Lowe's group at Cold Spring Harbor laboratory to do his postdoc research related to cellular senescence. http://www.cruk.cam.ac.uk/research-groups/narita-group 


\section{References}

1. Campisi, J. \& D'adda Di Fagagna, F. Cellular senescence: when bad things happen to good cells. Nat. Rev. Mol. Cell. Biol. 8, 729-740 (2007).

2. D'adda Di Fagagna, F. Living on a break: cellular senescence as a DNAdamage response. Nat. Rev. Cancer 8, 512-522 (2008).

3. Kuilman, T., Michaloglou, C., Mooi, W. J. \& Peeper, D. S. The essence of senescence. Gene. Dev. 24, 2463-2479 (2010).

4. Salama, R., Sadaie, M., Hoare, M. \& Narita, M. Cellular senescence and its effector programs. Gene. Dev. 28, 99-114 (2014).

5. Campisi, J. Aging, cellular senescence, and cancer. Annu. Rev. Physiol. 75, 685-705 (2013).

6. Hoare, M. \& Narita, M. Transmitting senescence to the cell neighbourhood. Nat. Cell Biol. 15, 887-889 (2013).

7. Hayflick, L. \& Moorhead, P. S. The serial cultivation of human diploid cell strains. Exp. Cell Res. 25, 585-621 (1961).

8. Hayflick, L. The limited in vitro lifetime of human diploid cell strains. Exp. Cell Res. 37, 614-636 (1965).

9. Harley, C. B., Futcher, A. B. \& Greider, C. W. Telomeres shorten during ageing of human fibroblasts. Nature 345, 458-460 (1990).

10. d'Adda di Fagagna, F. et al. A DNA damage checkpoint response in telomere-initiated senescence. Nature 426, 194-198 (2003).

11. Herbig, U., Jobling, W. A., Chen, B. P. C., Chen, D. J. \& Sedivy, J. M. Telomere shortening triggers senescence of human cells through a pathway involving ATM, p53, and p21(CIP1), but not p16(INK4a). Mol. Cell 14, 501513 (2004).

12. Blasco, M. A. Mice with bad ends: mouse models for the study of telomeres and telomerase in cancer and aging. EMBO J. 24, 1095-1103 (2005).

13. González-Suárez, E., Samper, E., Flores, J. M. \& Blasco, M. A. Telomerasedeficient mice with short telomeres are resistant to skin tumorigenesis. Nat. Genet. 26, 114-117 (2000).

14. Greenberg, R. A. et al. Short dysfunctional telomeres impair tumorigenesis in the INK4a(delta2/3) cancer-prone mouse. Cell 97, 515-525 (1999).

15. Qi, L. et al. Short telomeres and ataxia-telangiectasia mutated deficiency cooperatively increase telomere dysfunction and suppress tumorigenesis. Cancer Res. 63, 8188-8196 (2003).

16. Collado, M., Blasco, M. A. \& Serrano, M. Cellular senescence in cancer and aging. Cell 130, 223-233 (2007).

17. Feldser, D. M. \& Greider, C. W. Short telomeres limit tumor progression in vivo by inducing senescence. Cancer Cell 11, 461-469 (2007).

18. Rudolph, K. L. et al. Longevity, stress response, and cancer in aging telomerase-deficient mice. Cell 96, 701-712 (1999).

19. Serrano, M., Lin, A. W., McCurrach, M. E., Beach, D. \& Lowe, S. W. Oncogenic ras provokes premature cell senescence associated with accumulation of p53 and p16INK4a. Cell 88, 593-602 (1997). This seminal paper established the concept of OIS.

20. Lin, A. W. et al. Premature senescence involving p53 and p16 is activated in response to constitutive MEK/MAPK mitogenic signaling. Gene. Dev. 12, 
3008-3019 (1998).

21. Zhu, J., Woods, D., McMahon, M. \& Bishop, J. M. Senescence of human fibroblasts induced by oncogenic Raf. Gene. Dev. 12, 2997-3007 (1998).

22. Braig, M. et al. Oncogene-induced senescence as an initial barrier in lymphoma development. Nature 436, 660-665 (2005).

23. Chen, Z. et al. Crucial role of p53-dependent cellular senescence in suppression of Pten-deficient tumorigenesis. Nature 436, 725-730 (2005).

24. Collado, M. et al. Tumour biology: Senescence in premalignant tumours. Nature 436, 642-642 (2005).

25. Michaloglou, C. et al. BRAFE600-associated senescence-like cell cycle arrest of human naevi. Nature 436, 720-724 (2005).

This is a seminal study that identified OIS of human melanocytic naevi.

26. Lazzerini Denchi, E., Attwooll, C., Pasini, D. \& Helin, K. Deregulated E2F activity induces hyperplasia and senescence-like features in the mouse pituitary gland. Mol. Cell Biol. 25, 2660-2672 (2005).

27. Shamma, A. et al. Rb Regulates DNA damage response and cellular senescence through E2F-dependent suppression of N-ras isoprenylation. Cancer Cell 15, 255-269 (2009).

28. Courtois-Cox, S. et al. A negative feedback signaling network underlies oncogene-induced senescence. Cancer Cell 10, 459-472 (2006).

29. Gewinner, C. et al. Evidence that inositol polyphosphate 4-phosphatase type II is a tumor suppressor that inhibits PI3K signaling. Cancer Cell 16, 115125 (2009).

30. Chang, B. D. et al. A senescence-like phenotype distinguishes tumor cells that undergo terminal proliferation arrest after exposure to anticancer agents. Cancer Res. 59, 3761-3767 (1999).

31. Poele, te, R. H., Okorokov, A. L., Jardine, L., Cummings, J. \& Joel, S. P. DNA damage is able to induce senescence in tumor cells in vitro and in vivo. Cancer Res. 62, 1876-1883 (2002).

32. Schmitt, C. A. et al. A senescence program controlled by $\mathrm{p} 53$ and $\mathrm{p} 16 \mathrm{INK} 4 \mathrm{a}$ contributes to the outcome of cancer therapy. Cell 109, 335-346 (2002).

This was the first study showing that chemotherapy-induced tumour senescence contributed to the improved survival in mice, in an apoptosis defective context.

33. $\mathrm{Wu}, \mathrm{C} .-\mathrm{H}$. et al. Cellular senescence is an important mechanism of tumor regression upon c-Myc inactivation. Proc. Natl. Acad. Sci. USA 104, 1302813033 (2007).

34. Xue, W. et al. Senescence and tumour clearance is triggered by p53 restoration in murine liver carcinomas. Nature 445, 656-660 (2007).

This was the first study to show that the innate immune response eliminates the senescent cells induced in tumours in mice.

35. Ventura, A. et al. Restoration of p53 function leads to tumour regression in vivo. Nature 445, 661-665 (2007).

36. Reimann, M. et al. Tumor Stroma-Derived TGF-b Limits Myc-Driven Lymphomagenesisvia Suv39h1-Dependent Senescence. Cancer Cell 17, 262272 (2010).

37. Ewald, J. A., Desotelle, J. A., Wilding, G. \& Jarrard, D. F. Therapy-induced senescence in cancer. J. Natl. Cancer Inst. 102, 1536-1546 (2010).

38. Nardella, C., Clohessy, J. G., Alimonti, A. \& Pandolfi, P. P. Pro-senescence therapy for cancer treatment. Nat. Rev. Cancer 11, 503-511 (2011). 
39. Acosta, J. C. \& Gil, J. Senescence: a new weapon for cancertherapy. Trends Cell Biol. 22, 211-219 (2012).

40. Koppenol, W. H., Bounds, P. L. \& Dang, C. V. Otto Warburg's contributions to current concepts of cancer metabolism. Nat. Rev. Cancer 11, 325-337 (2011).

41. Kaplon, J. et al. A key role for mitochondrial gatekeeper pyruvate dehydrogenase in oncogene-induced senescence. Nature 498, 109-112 (2013). This study established the metabolomics profile of OIS cells, identifying active PDH with increased mitochondrial respiration during OIS.

42. Kondoh, H. et al. Glycolytic enzymes can modulate cellular life span. Cancer Res. 65, 177-185 (2005).

43. Dörr, J. R. et al. Synthetic lethal metabolic targeting of cellular senescence in cancer therapy. Nature 501, 421-425 (2013).

This study shows that in a TIS model the SASP, which provokes endoplasmic reticulum (ER) stress, is associated with increased glucose use, hyperactive mitochondrial respiration, and autophagy activation, and that TIS cells are sensitive to blocking glucose use or autophagy, which causes ER-related apoptosis.

44. Poulikakos, P. I. \& Rosen, N. Mutant BRAF melanomas--dependence and resistance. Cancer Cell 19, 11-15 (2011).

45. Bonnet, S. et al. A mitochondria-K+ channel axis is suppressed in cancer and its normalization promotes apoptosis and inhibits cancer growth. Cancer Cell 11, 37-51 (2007).

46. Michelakis, E. D., Webster, L. \& Mackey, J. R. Dichloroacetate (DCA) as a potential metabolic-targeting therapy for cancer. Br. J. Cancer 99, 989-994 (2008).

47. Haq, R. et al. Oncogenic BRAF regulates oxidative metabolism via PGC1 $\alpha$ and MITF. Cancer Cell 23, 302-315 (2013).

48. Vazquez, F. et al. PGC1 $\alpha$ expression defines a subset of human melanoma tumors with increased mitochondrial capacity and resistance to oxidative stress. Cancer Cell 23, 287-301 (2013).

49. St-Pierre, J. et al. Suppression of reactive oxygen species and neurodegeneration by the PGC-1 transcriptional coactivators. Cell 127, 397408 (2006).

50. Jiang, P., Du, W., Mancuso, A., Wellen, K. E. \& Yang, X. Reciprocal regulation of $\mathrm{p} 53$ and malic enzymes modulates metabolism and senescence. Nature 493, 689-693 (2013).

This study shows that Malic enzymes (MEs) and p53 reciprocally repress each other, and that depletion of ME1 or ME2 induces senescence in both normal and tumour cells.

51. Baggetto, L. G. Deviant energetic metabolism of glycolytic cancer cells. Biochimie 74, 959-974 (1992).

52. Ren, J.-G., Seth, P., Everett, P., Clish, C. B. \& Sukhatme, V. P. Induction of erythroid differentiation in human erythroleukemia cells by depletion of malic enzyme 2. PLoS ONE 5, e12520 (2010).

53. Quijano, C. et al. Oncogene-induced senescence results in marked metabolic and bioenergetic alterations. Cell Cycle 11, 1383-1392 (2012).

54. Passos, J. F., Saretzki, G. \& Zglinicki, Von, T. DNA damage in telomeres and mitochondria during cellular senescence: is there a connection? Nucleic Acids Res. 35, 7505-7513 (2007). 
55. Passos, J. F. et al. Mitochondrial dysfunction accounts for the stochastic heterogeneity in telomere-dependent senescence. Plos Biol. 5, e110 (2007).

56. López-Otín, C., Blasco, M. A., Partridge, L., Serrano, M. \& Kroemer, G. The hallmarks of aging. Cell 153, 1194-1217 (2013).

57. Moiseeva, O. et al. Mitochondrial dysfunction contributes to oncogeneinduced senescence. Mol. Cell Biol. 29, 4495-4507 (2009).

58. Narita, M. et al. Spatial Coupling of mTOR and Autophagy Augments Secretory Phenotypes. Science 332, 966-970 (2011).

59. Young, A. R. J. et al. Autophagy mediates the mitotic senescence transition. Gene. Dev. 23, 798-803 (2009).

This study shows that autophagy is activated during senescence, and that the inhibition of autophagy delays of onset of senescence and SASP.

60. Korolchuk, V. I. et al. Lysosomal positioning coordinates cellular nutrient responses. Nature 13, 453-460 (2011).

61. Guo, J. Y. et al. Activated Ras requires autophagy to maintain oxidative metabolism and tumorigenesis. Gene. Dev. 25, 460-470 (2011).

62. Wang, Y. et al. Autophagic activity dictates the cellular response to oncogenic RAS. Proc. Natl. Acad. Sci. 109, 13325-13330 (2012).

63. Tuveson, D. A. et al. Endogenous oncogenic K-ras(G12D) stimulates proliferation and widespread neoplastic and developmental defects. Cancer Cell 5, 375-387 (2004).

64. Mizushima, N. \& Komatsu, M. Autophagy: renovation of cells and tissues. Cell 147, 728-741 (2011).

65. Hoare, M., Young, A. R. J. \& Narita, M. Autophagy in cancer: Having your cake and eating it. Semin. Cancer Biol. 21, 397-404 (2011).

66. Yang, Z. J., Chee, C. E., Huang, S. \& Sinicrope, F. A. The role of autophagy in cancer: therapeutic implications. Mol. Cancer Ther. 10, 1533-1541 (2011).

67. Gewirtz, D. A. Autophagy and senescence in cancer therapy. J. Cell Physiol. 229, 6-9 (2014).

68. Amaravadi, R. K. et al. Autophagy inhibition enhances therapy-induced apoptosis in a Myc-induced model of lymphoma. J. Clin. Invest. 117, 326336 (2007).

69. Qu, X. et al. Promotion of tumorigenesis by heterozygous disruption of the beclin 1 autophagy gene. J. Clin. Invest. 112, 1809-1820 (2003).

70. Yue, Z., Jin, S., Yang, C., Levine, A. J. \& Heintz, N. Beclin 1, an autophagy gene essential for early embryonic development, is a haploinsufficient tumor suppressor. Proc. Natl. Acad. Sci. USA 100, 15077-15082 (2003).

71. Mariño, G. et al. Tissue-specific autophagy alterations and increased tumorigenesis in mice deficient in Atg4C/autophagin-3. J. Biol. Chem. 282, 18573-18583 (2007).

72. Takamura, A. et al. Autophagy-deficient mice develop multiple liver tumors. Gene. Dev. 25, 795-800 (2011).

73. Gewirtz, D. A. Autophagy and senescence: a partnership in search of definition. Autophagy 9, 808-812 (2013).

74. Lee, B. Y. et al. Senescence-associated beta-galactosidase is lysosomal betagalactosidase. Aging Cell 5, 187-195 (2006).

75. Gerland, L.-M. et al. Association of increased autophagic inclusions labeled for beta-galactosidase with fibroblastic aging. Exp. Gerontol. 38, 887-895 (2003).

76. Leidal, A. M., Cyr, D. P., Hill, R. J., Lee, P. W. K. \& McCormick, C. 
Subversion of autophagy by Kaposi's sarcoma-associated herpesvirus impairs oncogene-induced senescence. Cell Host Microbe 11, 167-180 (2012).

77. Mosieniak, G. et al. Curcumin induces permanent growth arrest of human colon cancer cells: link between senescence and autophagy. Mech. Ageing Dev. 133, 444-455 (2012).

78. Goehe, R. W. et al. The autophagy-senescence connection in chemotherapy: must tumor cells (self) eat before they sleep? J. Pharmacol. Exp. Ther. 343, 763-778 (2012).

79. Knizhnik, A. V. et al. Survival and death strategies in glioma cells: autophagy, senescence and apoptosis triggered by a single type of temozolomide-induced DNA damage. PLoS ONE 8, e55665 (2013).

80. Patel, K. R. et al. Sulfate metabolites provide an intracellular pool for resveratrol generation and induce autophagy with senescence. Science Translational Medicine 5, $205 \mathrm{ra} 133$ (2013).

81. Qi, M. et al. Pseudolaric acid B-induced autophagy contributes to senescence via enhancement of ROS generation and mitochondrial dysfunction in murine fibrosarcoma L929 cells. J. Pharmacol. Sci. 121, 200-211 (2013).

82. Qi, M. et al. mTOR inactivation by ROS-JNK-p53 pathway plays an essential role in Psedolaric acid B induced autophagy-dependent senescence in murine fibrosarcoma L929 cells. Eur. J. Pharmacol. 715, 76-88 (2013).

83. Patschan, S. et al. Lipid mediators of autophagy in stress-induced premature senescence of endothelial cells. Am. J. Physiol. Heart Circ. Physiol. 294, H1119-29 (2008).

84. Kang, H. T., Lee, K. B., Kim, S. Y., Choi, H. R. \& Park, S. C. Autophagy impairment induces premature senescence in primary human fibroblasts. PLOS ONE 6, e23367 (2011).

85. Janku, F., McConkey, D. J., Hong, D. S. \& Kurzrock, R. Autophagy as a target for anticancer therapy. Nat. Rev. Clin. Oncol. 8, 528-539 (2011).

86. Amaravadi, R. K. et al. Principles and current strategies for targeting autophagy for cancer treatment. Clin. Cancer Res. 17, 654-666 (2011).

87. Rosenfeldt, M. T. et al. p53 status determines the role of autophagy in pancreatic tumour development. Nature 504, 296-300 (2014).

88. Guo, J. Y. et al. Autophagy suppresses progression of K-ras-induced lung tumors to oncocytomas and maintains lipid homeostasis. Gene. Dev. 27, 1447-1461 (2013).

89. Kuilman, T. \& Peeper, D. S. Senescence-messaging secretome: SMS-ing cellular stress. Nat. Rev. Cancer 9, 81-94 (2009).

90. Coppé, J.-P., Desprez, P.-Y., Krtolica, A. \& Campisi, J. The senescenceassociated secretory phenotype: the dark side of tumor suppression. Annu. Rev. Pathol. 5, 99-118 (2010).

91. Kuilman, T. et al. Oncogene-Induced Senescence Relayed by an InterleukinDependent Inflammatory Network. Cell 133, 1019-1031 (2008).

92. Acosta, J. C. et al. Chemokine Signaling via the CXCR2 Receptor Reinforces Senescence. Cell 133, 1006-1018 (2008).

These studies ${ }^{91,92}$ describe the critical role of proinflammatory cytokines and their receptors in senescence.

93. Acosta, J. C. et al. A complex secretory program orchestrated by the inflammasome controls paracrine senescence. Nat. Cell Biol. 15, 978-990 (2013).

This study identified the inflammasome as a critical regulator of the 
SASP.

94. Lujambio, A. et al. Non-cell-autonomous tumor suppression by p53. Cell 153, 449-460 (2013).

95. Chien, Y. et al. Control of the senescence-associated secretory phenotype by NF- $\kappa \mathrm{B}$ promotes senescence and enhances chemosensitivity. Gene. Dev. 25, 2125-2136 (2011).

96. Rodier, F. et al. Persistent DNA damage signalling triggers senescenceassociated inflammatory cytokine secretion. Nature 11, 973-979 (2009).

97. Yoshimoto, S. et al. Obesity-induced gut microbial metabolite promotes liver cancer through senescence secretome. Nature 499, 97-101 (2013).

An interesting study showing a functional connection between obesityinduced senescence and stromal SASP.

98. Orjalo, A. V., Bhaumik, D., Gengler, B. K., Scott, G. K. \& Campisi, J. Cell surface-bound IL-1alpha is an upstream regulator of the senescenceassociated IL-6/IL-8 cytokine network. Proc. Natl. Acad. Sci. USA 106, 17031-17036 (2009).

99. Davalos, A. R. et al. p53-dependent release of Alarmin HMGB1 is a central mediator of senescent phenotypes. J. Cell Biol. 201, 613-629 (2013).

100. Millis, A. J., Hoyle, M., McCue, H. M. \& Martini, H. Differential expression of metalloproteinase and tissue inhibitor of metalloproteinase genes in aged human fibroblasts. Exp. Cell Res. 201, 373-379 (1992).

101. Goldstein, S., Moerman, E. J., Fujii, S. \& Sobel, B. E. Overexpression of plasminogen activator inhibitor type-1 in senescent fibroblasts from normal subjects and those with Werner syndrome. J. Cell Physiol. 161, 571-579 (1994).

102. Kortlever, R. M., Higgins, P. J. \& Bernards, R. Plasminogen activator inhibitor-1 is a critical downstream target of p53 in the induction of replicative senescence. Nat. Cell Biol. 8, 877-884 (2006).

103. Krtolica, A., Parrinello, S., Lockett, S., Desprez, P. Y. \& Campisi, J. Senescent fibroblasts promote epithelial cell growth and tumorigenesis: a link between cancer and aging. Proc. Natl. Acad. Sci. USA 98, 12072-12077 (2001).

104. Parrinello, S., Coppé, J.-P., Krtolica, A. \& Campisi, J. Stromal-epithelial interactions in aging and cancer: senescent fibroblasts alter epithelial cell differentiation. J. Cell Sci. 118, 485-496 (2005).

105. Coppé, J.-P. et al. Senescence-associated secretory phenotypes reveal cellnonautonomous functions of oncogenic RAS and the p53 tumor suppressor. Plos Biol 6, 2853-2868 (2008).

106. Hubackova, S. et al. IL1- and TGF $\beta$-Nox4 signaling, oxidative stress and DNA damage response are shared features of replicative, oncogene-induced, and drug-induced paracrine 'bystander senescence'. Aging (Albany NY) 4, 932-951 (2012).

107. Wajapeyee, N., Serra, R. W., Zhu, X., Mahalingam, M. \& Green, M. R. Oncogenic BRAF induces senescence and apoptosis through pathways mediated by the secreted protein IGFBP7. Cell 132, 363-374 (2008).

108. Scurr, L. L. et al. IGFBP7 is not required for B-RAF-induced melanocyte senescence. Cell 141, 717-727 (2010).

109. Wajapeyee, N., Serra, R. W., Zhu, X., Mahalingam, M. \& Green, M. R. Role for IGFBP7 in senescence induction by BRAF. Cell 141, 746-747 (2010).

110. Jackson, J. G. et al. p53-mediated senescence impairs the apoptotic response 
to chemotherapy and clinical outcome in breast cancer. Cancer Cell 21, 793806 (2012).

111. Hoenicke, L. \& Zender, L. Immune surveillance of senescent cells--biological significance in cancer- and non-cancer pathologies. Carcinogenesis 33, 11231126 (2012).

112. Kang, T.-W. et al. Senescence surveillance of pre-malignant hepatocytes limits liver cancer development. Nature 479, 547-551 (2011).

This is a seminal study identifying 'senescence-surveillance' involving the adaptive immune response.

113. Iannello, A., Thompson, T. W., Ardolino, M., Lowe, S. W. \& Raulet, D. H. p53-dependent chemokine production by senescent tumor cells supports

NKG2D-dependent tumor elimination by natural killer cells. J. Exp. Med. 210, 2057-2069 (2013).

This study suggests that NK cells recruited to the tumour lesion by the SASP of senescent tumour cells may also kill non-senescent tumour cells.

114. Raulet, D. H., Gasser, S., Gowen, B. G., Deng, W. \& Jung, H. Regulation of ligands for the NKG2D activating receptor. Annu. Rev. Immunol. 31, 413441 (2013).

115. Feig, C. et al. Targeting CXCL12 from FAP-expressing carcinoma-associated fibroblasts synergizes with anti-PD-L1 immunotherapy in pancreatic cancer.

Proc. Natl. Acad. Sci. USA 110, 20212-20217 (2013).

116. Rakhra, K. et al. CD4(+) T cells contribute to the remodeling of the microenvironment required for sustained tumor regression upon oncogene inactivation. Cancer Cell 18, 485-498 (2010).

117. Willimsky, G. \& Blankenstein, T. Sporadic immunogenic tumours avoid destruction by inducing T-cell tolerance. Nature 437, 141-146 (2005).

118. Guerra, C. et al. Pancreatitis-induced inflammation contributes to pancreatic cancer by inhibiting oncogene-induced senescence. Cancer Cell 19, 728-739 (2011).

119. Bavik, C. et al. The gene expression program of prostate fibroblast senescence modulates neoplastic epithelial cell proliferation through paracrine mechanisms. Cancer Res. 66, 794-802 (2006).

120. Krizhanovsky, V. et al. Senescence of activated stellate cells limits liver fibrosis. Cell 134, 657-667 (2008).

This was the first study to show that hepatic stellate cell senescence controls fibrosis through the SASP.

121. Friedman, S. L. Hepatic stellate cells: protean, multifunctional, and enigmatic cells of the liver. Physiol. Rev. 88, 125-172 (2008).

122. Kim, K.-H., Chen, C.-C., Monzon, R. I. \& Lau, L. F. Matricellular protein CCN1 promotes regression of liver fibrosis through induction of cellular senescence in hepatic myofibroblasts. Mol. Cell Biol. 33, 2078-2090 (2013).

123. Jun, J.-I. \& Lau, L. F. The matricellular protein CCN1 induces fibroblast senescence and restricts fibrosis in cutaneous wound healing. Nat. Cell Biol. 12, 676-685 (2010).

124. Burd, C. E. et al. Monitoring tumorigenesis and senescence in vivo with a p16(INK4a)-luciferase model. Cell 152, 340-351 (2013).

This study shows a close and general correlation between stromal senescence and early neoplastic events in a new p16-monitor mouse model.

125. Yang, G. et al. The chemokine growth-regulated oncogene 1 (Gro-1) links 
RAS signaling to the senescence of stromal fibroblasts and ovarian tumorigenesis. Proc. Natl. Acad. Sci. USA 103, 16472-16477 (2006).

126. Braumüller, H. et al. T-helper-1-cell cytokines drive cancer into senescence. Nature 494, 361-365 (2013).

This study shows that tumours can be induced into senescence by cytokines derived from tumour associated antigen (TAA)-activated CD4+ Th1 cells.

127. Bergers, G., Javaherian, K., Lo, K. M., Folkman, J. \& Hanahan, D. Effects of angiogenesis inhibitors on multistage carcinogenesis in mice. Science 284, 808-812 (1999).

128. Müller-Hermelink, N. et al. TNFR1 signaling and IFN-gamma signaling determine whether $\mathrm{T}$ cells induce tumor dormancy or promote multistage carcinogenesis. Cancer Cell 13, 507-518 (2008).

129. Casanovas, O., Hager, J. H., Chun, M. G. H. \& Hanahan, D. Incomplete inhibition of the $\mathrm{Rb}$ tumor suppressor pathway in the context of inactivated p53 is sufficient for pancreatic islet tumorigenesis. Oncogene 24, 6597-6604 (2005).

130. Quesnel, B. Tumor dormancy and immunoescape. APMIS 116, 685-694 (2008).

131. Lengagne, R. et al. Distinct role for CD8 T cells toward cutaneous tumors and visceral metastases. J. Immunol. 180, 130-137 (2008).

132. Eyles, J. et al. Tumor cells disseminate early, but immunosurveillance limits metastatic outgrowth, in a mouse model of melanoma. J. Clin. Invest. 120, 2030-2039 (2010).

133. Elinav, E. et al. Inflammation-induced cancer: crosstalk between tumours, immune cells and microorganisms. Nat. Rev. Cancer 13, 759-771 (2013).

134. Nickoloff, B. J., Ben-Neriah, Y. \& Pikarsky, E. Inflammation and cancer: is the link as simple as we think? J. Invest. Dermatol. 124, x-xiv (2005).

135. Nickoloff, B. J. Creation of psoriatic plaques: the ultimate tumor suppressor pathway. A new model for an ancient T-cell-mediated skin disease.

Viewpoint. J. Cutan. Pathol. 28, 57-64 (2001).

136. Nickoloff, B. J. \& Nestle, F. O. Recent insights into the immunopathogenesis of psoriasis provide new therapeutic opportunities. J. Clin. Invest. 113, 16641675 (2004).

137. Kaelin, W. G. \& McKnight, S. L. Influence of metabolism on epigenetics and disease. Cell 153, 56-69 (2013).

138. Lu, C. \& Thompson, C. B. Metabolic regulation of epigenetics. Cell Metab. 16, 9-17 (2012).

139. Draoui, N. \& Feron, O. Lactate shuttles at a glance: from physiological paradigms to anti-cancer treatments. Dis. Model Mech. 4, 727-732 (2011).

140. Giancotti, F. G. Mechanisms governing metastatic dormancy and reactivation. Cell 155, 750-764 (2013).

141. Martins, C. P., Brown-Swigart, L. \& Evan, G. I. Modeling the therapeutic efficacy of p53 restoration in tumors. Cell 127, 1323-1334 (2006).

142. Dickins, R. A. et al. Tissue-specific and reversible RNA interference in transgenic mice. Nat. Genet. 39, 914-921 (2007).

143. Pribluda, A. et al. A senescence-inflammatory switch from cancer-inhibitory to cancer-promoting mechanism. Cancer Cell 24, 242-256 (2013).

144. Cristofalo, V. J., Lorenzini, A., Allen, R. G., Torres, C. \& Tresini, M. Replicative senescence: a critical review. Mech. Ageing Dev. 125, 827-848 
(2004).

145. Sharpless, N. E. \& Depinho, R. A. How stem cells age and why this makes us grow old. Nat Rev Mol Cell Biol 8, 703-713 (2007).

146. Baker, D. J. et al. Opposing roles for p16Ink4a and p19Arf in senescence and ageing caused by BubR1 insufficiency. Nature 10, 825-836 (2008).

147. Sousa-Victor, P. et al. Geriatric muscle stem cells switch reversible quiescence into senescence. Nature 506, 316-321 (2014).

148. Baker, D. J. et al. Clearance of p16Ink4a-positive senescent cells delays ageing-associated disorders. Nature 479, 232-236 (2012).

149. Muñoz-Espín, D. et al. Programmed cell senescence during mammalian embryonic development. Cell 155, 1104-1118 (2013).

150. Storer, M. et al. Senescence is a developmental mechanism that contributes to embryonic growth and patterning. Cell 155, 1119-1130 (2013).

151. Chuprin, A. et al. Cell fusion induced by ERVWE1 or measles virus causes cellular senescence. Gene. Dev. 27, 2356-2366 (2013).

152. Alimonti, A. et al. A novel type of cellular senescence that can be enhanced in mouse models and human tumor xenografts to suppress prostate tumorigenesis. J. Clin. Invest. 120, 681-693 (2010).

153. Schlomm, T. et al. Clinical significance of p53 alterations in surgically treated prostate cancers. Mod. Pathol. 21, 1371-1378 (2008).

154. Song, M. S., Salmena, L. \& Pandolfi, P. P. The functions and regulation of the PTEN tumour suppressor. Nat. Rev. Mol. Cell Biol. 13, 283-296 (2012).

155. Soucek, L. et al. Modelling Myc inhibition as a cancer therapy. Nature 455, 679-683 (2008).

156. Lin, H.-K. et al. Skp2 targeting suppresses tumorigenesis by Arf-p53independent cellular senescence. Nature 464, 374-379 (2010).

157. Campaner, S. et al. Cdk2 suppresses cellular senescence induced by the cmyc oncogene. Nature 12, 54-9 (2010).

158. Puyol, M. et al. A synthetic lethal interaction between K-Ras oncogenes and Cdk4 unveils a therapeutic strategy for non-small cell lung carcinoma.

Cancer Cell 18, 63-73 (2010).

159. Chan, C.-H. et al. Pharmacological inactivation of Skp2 SCF ubiquitin ligase restricts cancer stem cell traits and cancer progression. Cell 154, 556-568 (2013).

160. Wall, M. et al. The mTORC1 inhibitor everolimus prevents and treats E $\mu$ Myc lymphoma by restoring oncogene-induced senescence. Cancer Discov. 3, 82-95 (2013). 


\begin{tabular}{|c|c|c|c|c|}
\hline Approach & Genetic background & Senescence inducer & Tumour phenotype & References \\
\hline \multicolumn{5}{|c|}{ Molecular induction of senescence } \\
\hline \multirow{3}{*}{ Trp53 activity restoration } & LSL-Trp53; Rosa26-Cre-ERT2 & Endogenous p53 re-expression & Regression of sarcomas & 35 \\
\hline & 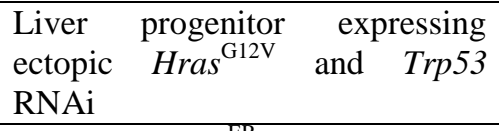 & Endogenous p53 re-expression & Regression of liver tumours & 34 \\
\hline & $\mathrm{E} \mu-M y c ; \operatorname{Trp} 53^{\mathrm{ER}} ;$ ectopic $B c l 2$ & p53 reactivation & Senescence induction in lymphoma & 36 \\
\hline Telomerase dysfunction & E $\mu-M y c ;$ ectopic $B c l 2$ & mTR ablation & Suppression of Burkitt's lymphoma & 17 \\
\hline \multirow[b]{4}{*}{ Oncogene inactivation } & Inducible $M Y C$ allele & MYC suppression & $\begin{array}{l}\text { Suppression of lymphoma, osteosarcoma, and } \\
\text { hepatocellular carcinoma }\end{array}$ & 33 \\
\hline & $\begin{array}{l}\text { LSL-Kras }{ }^{\mathrm{G} 12 \mathrm{D}} ; \\
\text { infection }\end{array}$ & Omo-myc expression & Regression of lung tumours & 155 \\
\hline & $\begin{array}{l}\text { Inducible MYC allele in } \\
\text { haematopoietic system }\end{array}$ & MYC suppression & $\begin{array}{l}\text { Regression of } \mathrm{T} \text { cell acute lymphoblastic } \\
\text { lymphoma }\end{array}$ & 116 \\
\hline & $\begin{array}{lll}\text { Inducible } B C R-A B L & \text { allele } & \text { in } \\
\text { hematopoietic system } & & \\
\end{array}$ & BCR-ABL suppression & Regression of pro-B cell leukemia & 116 \\
\hline \multirow[b]{4}{*}{ Cell cycle deregulation } & $\mathrm{Pten}^{+/-}$ & Skp2 ablation & $\begin{array}{l}\text { Lymphadenopathy and adrenal tumour } \\
\text { abrogation }\end{array}$ & 156 \\
\hline & Pten ${ }^{\operatorname{loxP} / 10 x P} ; \mathrm{Pb}-\mathrm{Cre}$ & Skp2 ablation & Prostate cancer restriction & 156 \\
\hline & $\mathrm{E} \mu-M y c$ & $C d k 2$ ablation & Lymphomagenesis delay & 157 \\
\hline & LSL-Kras $^{\mathrm{G} 12 \mathrm{~V}} ; \mathrm{RERTn}^{+/ \mathrm{ERT}}$ & Cdk4 ablation & $\begin{array}{l}\text { Suppression of non-small cell lung carcinoma } \\
\text { development }\end{array}$ & 158 \\
\hline \multicolumn{5}{|c|}{ Pharmacological-induction of senescence } \\
\hline DNA damage & E $\mu-M y c ;$ ectopic $B c l 2$ & cyclophosphamide & Lymphoma regression & 32,43 \\
\hline DNA damage & E $\mu-N \operatorname{ras}^{\mathrm{G}}{ }^{2 \mathrm{~V}} ;$ ectopic $\mathrm{Bcl} 2$ & Doxorubicin & Lymphoma regression & 22 \\
\hline PTEN inhibitor & Human prostate cancer xenograft & VO-OHpic [ & Tumour growth inhibition & 152 \\
\hline MDM2 inhibitor & Pten $^{\text {loxP/oxP }} ; \mathrm{Pb}-\mathrm{Cre}$ & Nutlin-3 & Prostate tumour size decreased & 152 \\
\hline CDK4 inhibitor & $\mathrm{LSL}_{-K r a s}{ }^{\mathrm{G} 12 \mathrm{~V}} ; \mathrm{RERTn}^{+/ \mathrm{ERT}}$ & PD0332991 & $\begin{array}{l}\text { Suppression of non-small cell lung carcinoma } \\
\text { development }\end{array}$ & 158 \\
\hline SKP2 inhibitor & $\begin{array}{l}\text { Human lung and prostate cancer } \\
\text { xenografts }\end{array}$ & SKP2 Inhibitor\#25 & Tumour growth inhibition & 159 \\
\hline mTOR inhibitor & $\mathrm{E} \mu-M y c$ & Everolimus & Lymphoma regression & 160 \\
\hline \multicolumn{5}{|c|}{ Immunotherapeutic-induction of senescence } \\
\hline $\mathrm{T}_{\mathrm{H}} 1$ cell cytokines & RIP-Tag2 & IFN $\gamma$ and TNF & Pancreatic $\beta$-cell tumour regression & 126 \\
\hline
\end{tabular}

Table 1: Therapy-Induced Senescence Strategies in vivo

LSL: LoxP-STOP-LoxP.

$\operatorname{Tr} 53 E R$ : knockin allele, composed of endogenous $\operatorname{Trp} 53$ fused with the sequence encoding a modified ligand-binding domain of the mouse 
estrogen receptor (ER).

VO-OHpic: Hydroxy(oxo)vanadium 3-hydroxypyridine-2-carboxylic acid trihydrate.

$m T R$ : mouse telomerase RNA component (also known as Terc). 